\title{
Integration of system for research in health with computation in cloud using artificial intelligence
}

\begin{abstract}
Currently the use of information technology is often applied in the health area. With regard to scientific research, we have the SINPEC Integrated System of Electronic Protocols. The purpose of this tool is to support the researcher in this area who until now did not have statistical tests. The main objective of this work is to provide SINPEC users with the improvement in health data analysis through the use of cognitive computing technologies.
\end{abstract}

Keywords: artificial intelligence, cloud computing, information systems
Volume 7 Issue 4 - 2018

\section{Carlos Henrique Kuretzki,' José Simão de Paula Pinto, ${ }^{2}$ José Claudio Vianna ${ }^{3}$ \\ 'Professor of the Program for Analysis and Development of Systems and Computer Engineering, Federal University, Brazil ${ }^{2}$ Professor of the Information Management Program, Federal University, Brazil \\ ${ }^{3}$ Professor of the Computer Engineering, Universidade Positivo,} Brazil

\author{
Correspondence: Carlos Henrique Kuretzki, Professor of \\ the Program for Analysis and Development of Systems and \\ Computer Engineering, Federal University, Brazil, \\ Email carlos@carlos.pro.br
}

Received: June 29, 2018 | Published: August 08, 2018

\section{Introduction}

The health sector is one of the largest in most countries and can significantly benefit from high-quality, real-time and locationindependent data. However, many healthcare professionals are not familiar with information technology solutions, business and information or are reluctant to apply them in their work area. The term Medical Informatics originated in the 1970s, inspired by the French word for Computer Science (informatique) thus passing the English-speaking community to adopt the Medical Informatics form; previously the term related to biomedical research was vaguely defined. During the 1980s in Europe this term became broader than Medical Computing, including subjects such as Medical Statistics, Record Keeping, as well as others related to Medical Informatics itself. Over the years the word "medical" applied to this terminology gave a restrictive connotation of application to the medical field, thus, being replaced by the word health, leading to Health Informatics. ${ }^{1}$ Nowadays there are many areas that make use of technologies based on Health Informatics. Solutions that can give equal or better results than those provided by professionals. These solutions are called specialized systems. The main purpose of these systems is to find a solution to problems that are usually solved by specialized professionals in the field, and therefore the system is able to decide on its own, only based on specific and selected knowledge. This knowledge is given by the skilled professionals and based on real cases. ${ }^{2}$ Cognitive computing systems are perceptual-sensitive systems that react to external signals and learn to do something with excellence through data analysis. That is, they receive numerous unstructured data entries and process them in order to obtain information in response to a given question or task. Cognitive technology, as a rule, proposes to perform tasks that only humans have so far been able to do, is only supposed to do it faster and more accurately.

Cognitive solutions may comprise different types of data, such as laboratory values in a structured database or text as scientific publication. Cognitive solutions are trained to understand industryspecific technical content and use advanced thinking, predictive modeling, and machine learning techniques to advance research more quickly. ${ }^{4}$ IBM Watson is a cognitive computing technology and have been used to investigate the biological and health sciences. It is based on medical literature, patents, genetic, and chemical and pharmacological data. ${ }^{5}$ The IBM's Watson platform combines some capabilities, building a complete cognitive system, which is being called the new era of computing. These capabilities are: Natural language processing, which helps you understand the complexity of unstructured data, which are currently more than $80 \%$ of the data in the world; the generation and evaluation of hypotheses, using advanced analysis to weigh and evaluate a quantity of answers based on relevance, dynamic learning, which helps improve learning based on outputs, becoming smarter with each interaction. ${ }^{6}$ With regard to the use of technology for scientific research in health, we have the SINPEC Integrated System of electronic Protocols, a tool that by purpose supports the health researcher in the conduct of his research. The objective of this article is to define the architecture for integration of SINPE( ) with artificial intelligence APIs in cloud computing.

\section{Justification}

Today the use of cognitive computing technologies is very common in the health area. As they can understand, reason, and learn, they are helping people to expand their knowledge base, improve their productivity, and extend their expertise. The field of health care is been substantially getting deepen because, with cognitive computing, professionals are now able to see health data that were previously inaccessible. In view of this evolution, it is intended to stimulate researchers using SINPE@ the use of this technology, as well as to obtain the results of the data analysis of IBM Watson that is highlighting with electronic health records.

\section{Materials and methods}

To carry out this research, it will be necessary to define the medical center where the work will be applied, as well as the sample of clinical records for the research. In this context, it is possible to perform the research at the IPO Hospital in Curitiba. Center of reference in the region in otorhinolaryngology. After this definition, will be submitted to the research ethics committee of the institution, evaluation of this 
research without contact with patient for deliberation and publication of the work in the Brazilian Platform. The IPO Hospital has scientific research using SINPEC with a focus on otorhinolaryngology. In addition to these databases containing clinical research in otorhinolaryngology, since 2005 SINPEC has research in other health specialties and also in management. Currently having research in digestive surgery, ophthalmology, bariatric surgery and others. It is also intended to conduct the academic week of information management lecture regarding the research. Then, the data analysis using the mentioned technologies starts and the partial results are presented in the research seminar in the postgraduate program. In the intermediate phase of this research will be proposed extension course on artificial intelligence, cognitive computing and/or machine learning

\section{Discussion}

The IBM Watson Health was launched in April 2015, with the mission to improve the ability of professionals from the health area and innovating by searching for insights from a massive amount of data. IBM has gathered data by partnered with various academic, research, and private institutions. The objective was to apply Watson and cognitive computing to data of many various treatments. Also, Watson is helping doctors to find clinical trials for different patients, identifying the most efficient treatment and matching them. ${ }^{7}$ When an electronic health record is available (EHR), the data contained in it demand from physicians to be responsible for more information as the medical records are assimilated by multiple institutions and systems. If not designed in accordance with cognitive considerations, the technology may become a deterrent to patient care. Considering that, to promote physician adoption and successfully use of the EHR, it is needed ways for searching the correcting information within a patient record. In shows a study to identify whether using a semantic search would improve a physician's time to find information as well as the accuracy of a particular diagnosis. ${ }^{8}$ Usually, healthcare systems are divided into three layers: collection layer, transmission layer and analysis layer. And although theses system have been bringing improvement for the health care area, there are known problems that difficult its uses, like medical multi-modality data, network inflexibility and communication latency. To solve theses problems,, 10 proposes a Edge-Cognitive-Computing-based (ECC-based) smart healthcare system, that can monitor and analyze users physical health, obtaining the corresponding health risk grade of a user based on it's status, and solving the resource allocation and switching problems of the edge cloud based on the user's physical health cognition. After studying the technologies, they opted to use the following systemic architecture to promote this integration:

\section{Programming language: Microsoft .Net and C\# \\ Database: Microsoft SQL Server}

Hosting: Infrastructure-like service (IaaS) with Amazon AWS.

As future work intends to apply this architecture to first integrate Watson's cognitive computing solutions and later with other suppliers such as Azure and Amazon AWS. Health care is usually provided by diagnostic and treatment selections made by an average of patients. By doing this, it is well known that the results can vary widely among different populations. It is necessarily to incorporate more information about individual patients into each medical decision, so that medical professionals can make the best choice for each patient, rather than an average. Cognitive computing provides this pathway for more individualized medical decisions, meaning that health professionals may be able to offer more patient care to patients with a greater diagnostic safety. ${ }^{11}$

\section{Conclusion}

With the implementation of this software architecture, users using the SINPEC research software to conduct their health research will be able to use the main cognitive computing technologies in the cloud. Figure 1 It is also intended that these users can use these technologies even if their base area is not technological, potentiating results for the health area and related researchers. ${ }^{12}$ This implementation will enable faster discoveries and results in health by integrating the electronic record of patients with cognitive computing providers.

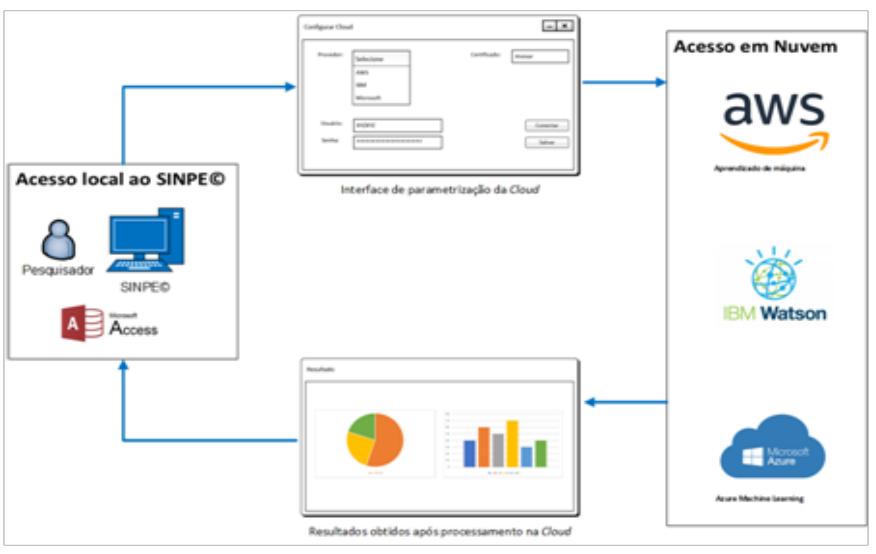

Figure I Proposed architecture for integration.

\section{Acknowledgments}

None.

\section{Conflict of interests}

The author declares no conflict of interest.

\section{References}

1. Cunha H, Ribeiro S. Introdução Aos Sistemas Especialistas. Livros Técnicos E Científicos; 1987

2. Chen Y, Argentinis JDE, Weber G. Ibm Watson: How Cognitive Computing Can Be Applied To Big Data Challenges In Life Sciences Research. Clinical Therapeutics. 2016;38(4):688-701.

3. Efraim T, Volonino L. Tecnologia Da InformaçÃo Para Gestão. 8th edn. Bookman, USA; 2013.

4. Sabbatini RME. A Telemedicina No Brasil: Evolução E Perspectivas. In: Informatica Em Saúde: Uma Perspectiva Multiprofissional Dos Usos E Possibilidades. São Caetano Do Sul Yendis. 2012;1-16.

5. Shader RI. Some Reflections on Ibm Watson and on Women's Health. Clinical Therapeutics. 2016;38(1):1-2.

6. Shortliffe EH, Cimino JJ. Biomedical Informatics: The Science and The Pragmatics. In: Biomedical Informatics Computer. Applications In Health Care And Biomedicine; 2013.

7. Ratner M. Ibm's Watson Group Signs Up Genomics Partners. Nature Biotechnology. 2015;33(1):10-11. 
8. Chen Y, Elenee Argentinis J, Weber G. Ibm Watson: How Cognitive Computing Can Be Applied To Big Data Challenges In Life Sciences Research. Clinical Therapeutics. 2016;38(4):688-701.

9. High R. The Era Of Cognitive Systems: An Inside Look At Ibm Watson and How It Works. Redguides For Business Leaders. 2012;1(1):1-14.

10. Ahmed MN, Toor AS, O'neil K, et al. Cognitive Computing And The Future of Health Care Cognitive Computing And The Future of Healthcare: The Cognitive Power of Ibm Watson Has The Potential To Transform Global Personalized Medicine. Ieee Pulse. 2017;8(3):4-9.
11. Tawfik AA, Kochendorfer KM, Saparova D, et al. Using Semantic Search To Reduce Cognitive Load In An Electronic Health Record. Ieee 13th International Conference on E-Health Networking. Applications And Services. 2011;181-184.

12. Chen M, Li W, Hao Y, et al. Edge Cognitive Computing Based Smart Healthcare System. Future Generation Computer Systems. 2018;86:403-411. 